\title{
PARALLEL KLYSTRON OPERATION AT THE ADVANCED PHOTON SOURCE STORAGE RING*
}

\author{
Doug Horan $\dagger$ and Ernest Cherbak \\ Advanced Photon Source, Argonne National Laboratory, Argonne, IL
}

\begin{abstract}
A parallel-klystron topology has been designed and tested at the Advanced Photon Source to supply rf power to the $7-\mathrm{GeV}$ storage ring rf cavities. The rf power output of two 1.1-MW, $352-\mathrm{MHz}$ rf stations is combined utilizing a 3-dB/90-degree hybrid. The two outputs of the hybrid then supply rf power to two sectors of storage-ring cavities. Feedback control loops maintain the proper output phase and amplitude from each rf station at the combining hybrid input ports so that the correct power balance and phase relationship is maintained between the two sectors of storage ring cavities. Downstream mechanical phase shifters correct for phase changes when only one of the two rf stations is used in single-ended mode. EPICS is utilized to monitor and control the parallel-klystron system.
\end{abstract}

\section{INTRODUCTION - APS 352 MHZ RF SYSTEM TOPOLOGY}

The Advanced Photon Source (APS) storage ring rf systems operate at a nominal rf frequency of $351.93 \mathrm{MHz}$ and supply the necessary rf power to store up to $300 \mathrm{~mA}$ of electrons at $7 \mathrm{GeV}$. There are four 1.1-MW klystronbased continuous-wave (CW) rf systems dedicated to supply power to the 16 storage ring rf cavities. A waveguide switching/ combining system (see Fig. 1) is utilized to direct and combine the outputs of the four $\mathrm{rf}$ systems to provide 12 discrete operating modes [1]. These modes allow single-ended or parallel operation of the storage-ring rf systems. Parallel-klystron operation will be required to store more than $150 \mathrm{~mA}$ in the APS storage ring because the total amount of rf power required will exceed 2 MW CW. The 16 single-cell rf cavities in the storage ring are grouped in four sectors of four cavities each. These four sectors are combined in pairs, with each pair being driven by either a single rf station or two rf stations operating in parallel. In both cases, WR2300, 3-dB hybrids, are used as splitters in singleended modes, or as a combiner/splitters in parallel modes. Motor-driven WR2300 phase shifters located after the hybrid output ports compensate for output phase changes dependent on whether the hybrids are being driven in single-ended or parallel fashion. The phase of the rf power driving the storage ring cavity sectors must be kept within approximately 5 degrees of optimum for stable beam operation, and the off power equally divided between the sector-pairs to avoid stressing cavity components. Using 3 -dB, 90-degree hybrids to combine and then equally split the output of two rf systems requires that the output amplitude and phase of the parallel rf systems be controlled to within $6 \%$ and 2 degrees, respectively. Lowlevel rf and high-voltage power supply (HVPS) controls are utilized to maintain these amplitude and phase tolerances.

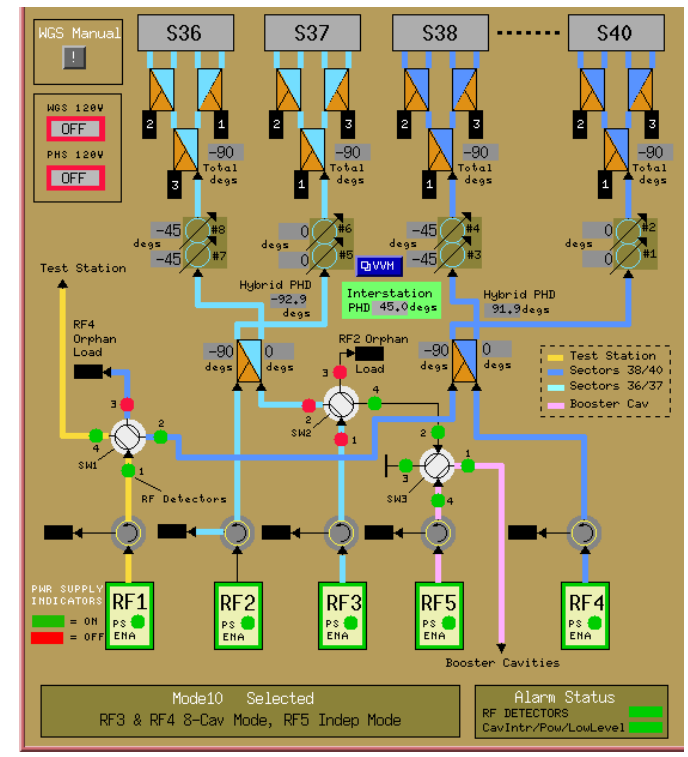

Figure 1: APS rf waveguide switching and combining system.

\section{PARALLEL-KLYSTRON PHASE AND AMPLITUDE CONTROL}

A block diagram of the APS parallel-klystron configuration is shown in Fig. 2. The rf power output from two $1.1-\mathrm{MW}, 352-\mathrm{MHz}$ rf systems is combined and then split by a 3-dB, 90-degree WR2300 waveguide hybrid. The two outputs of the hybrid each supply onehalf of the combined rf power to one sector of rf cavities. Directional couplers installed in the straight waveguide sections just prior to the hybrid ports sample the signals at each port of the hybrid (see Fig. 3). These directional couplers are all positioned at precisely the same distance from each hybrid port waveguide flange to establish accurate phase references. Low-loss coaxial cables, each trimmed to equal phase length within $+/-1$ degree at 352 $\mathrm{MHz}$, are used to route the outputs of these directional couplers to the low-level rf (LLRF) systems at each rf station. The output signals from the hybrid-input directional couplers are each split four ways in identical fashion to provide numerous phase-equalized signal sources for LLRF controls.

\footnotetext{
*Work supported by the U.S. Department of Energy, Office of Basic Energy Sciences, under Contract No. W-31-109-ENG-38.

†horan@aps.anl.gov
} 


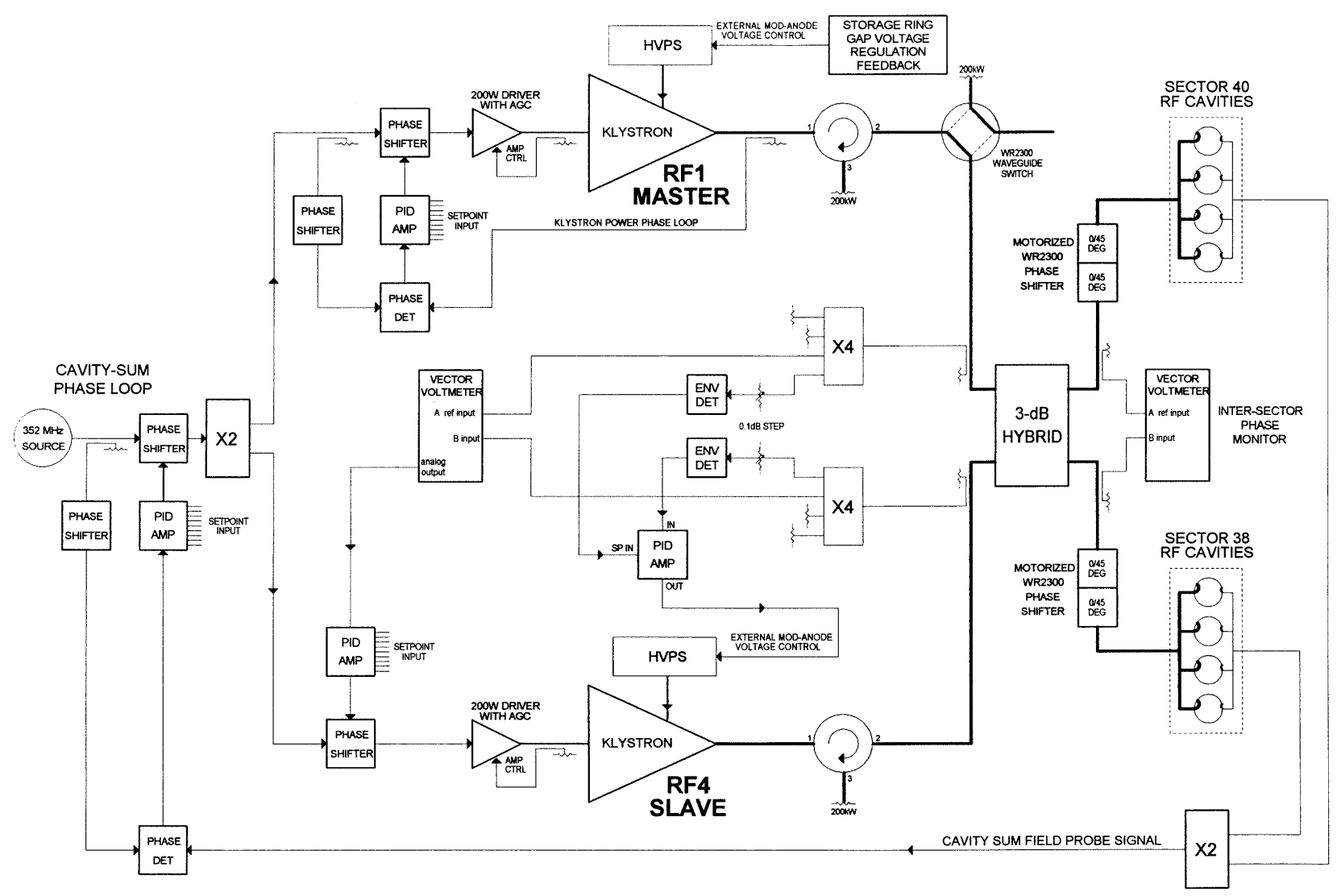

Figure 2: APS parallel-klystron configuration block diagram.

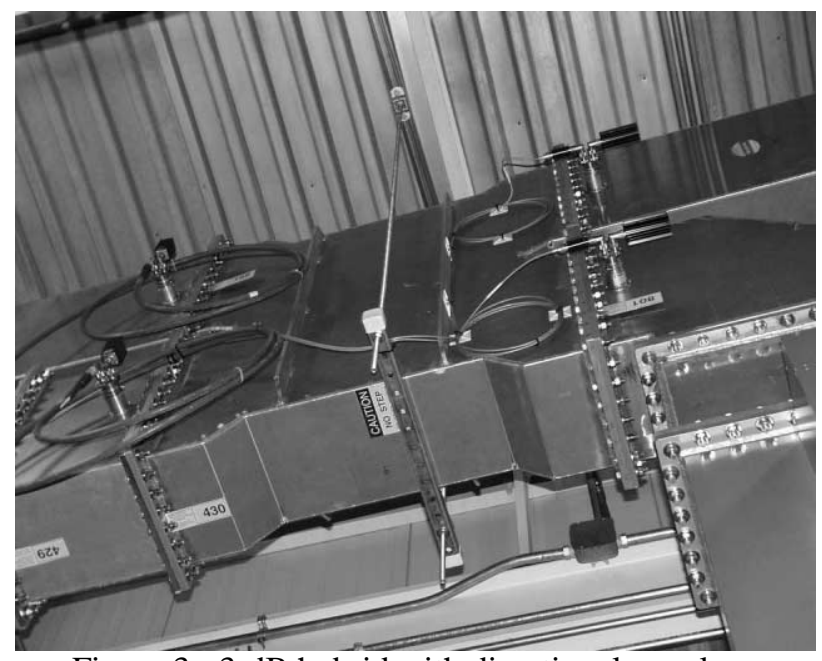

Figure 3: 3-dB hybrid with directional couplers.

The two rf stations are operated in parallel configuration utilizing a "master-slave" relationship. As shown in Figs. 1 and 2, RF1 is the "master" station and RF4 is the "slave" station. The LLRF controls maintain a constant phase and amplitude relationship between the two stations at the inputs of the hybrid, thereby maintaining a constant relationship between amplitude and phase, respectively, at the output ports of the hybrid. The master station provides both the phase and amplitude references for the slave station. The phase-control loop maintains a constant phase relationship between the two stations at the input of the hybrid, and consists of an HP8508A vector voltmeter utilized as a phase detector in direct analog-out mode, a proportional-integraldifferential (PID) amplifier, and an electronic phase shifter in the rf driveline of the slave station. A vector voltmeter is used as a phase detector to minimize amplitude-to-phase cross-modulation, with the reference input of the voltmeter driven by the output of the master station. The setpoint of the phase-control PID card is manually adjusted to maintain power balance between the hybrid output ports.

The amplitude-control loop maintains a constant amplitude relationship between the two stations at the input of the hybrid, utilizing two envelope detectors and a PID amplifier to generate a slave-station amplitude control voltage. The sampled output of the master station is envelope-detected then applied to the setpoint input of the amplitude-loop PID amplifier, establishing the reference level for the amplitude-control loop. The sampled output of the slave station is also envelopedetected then applied to the input of the amplitude-loop PID amplifier to provide the readback signal for the loop. The output of the PID amplifier is then used to control the mod-anode voltage of the slave-station HVPS and thereby amplitude-modulate the klystron rf output power, making whatever changes in klystron mod-anode voltage that are necessary to maintain lock of the amplitude-control loop. Mod-anode voltage control is also used to control the rf 
output of the master station for regulation of the storagering gap voltage in response to beam loading effects, and is applied to all storage-ring rf stations individually when they are operated in single-ended mode [2]. Remotecontrolled variable attenuators, with a range of $1.5 \mathrm{~dB}$ in $0.1 \mathrm{~dB}$ steps, are located immediately prior to the inputs of the envelope detectors to provide a manual offset adjustment point for the power balance between the stations. Since the power balance at the input ports of the hybrid affects the phase difference between the output ports, these variable attenuators are used to adjust the relative phase relationship between the output ports of the hybrid. A second HP8505A vector voltmeter is used to monitor the phase and amplitude of the hybrid output port signals to provide a readback of system performance locally, and remotely by network connections to the Experimental Physics and Industrial Control System (EPICS).

Fast rf interlocking is used to prevent over-powering of the rf cavities, should the outputs of the two parallel rf stations drift appreciatively out of phase from one another at the input ports of the combining/splitting hybrid. A power monitor chassis is utilized to detect the level of field probe signal coming from each cavity. If this calibrated rf level exceeds the equivalent of $2.3 \mathrm{~dB}$ above the nominal injection power level of approximately $40 \mathrm{kw} /$ cavity, the power monitor system will interrupt the low-level rf drive to the klystron driver amplifiers within $100 \mu \mathrm{s}$.

A cavity-sum phase loop is an integral part of each storage-ring rf station, and is used to stabilize and control cavity gap-voltage phase referenced to the common rf source signal driving all four stations. When the stations are operated in single-ended mode, the individual cavitysum phase loops are utilized for this purpose. When two stations are operated in parallel-mode, the master-station cavity-sum loop is utilized to provide phase-corrected $\mathrm{rf}$ drive to both stations. Coaxial rf relays controlled by a programmable logic controller are used to automatically configure the cavity-sum phase loop signals depending on the operating mode desired.

\section{SYSTEM PERFORMANCE WITH STORED BEAM}

Initial no-beam tests of the parallel-klystron operating mode were performed in August 1999. The RF1 and RF4 stations were operated in parallel, driving cavity sectors 38 and 40, with the RF2 station operating in single-ended mode driving cavity sectors 36 and 37 . The tests demonstrated that the gap-voltage phase stability of the paralleled rf stations met the 1-degree peak-to-peak specification for the APS rf systems, with a static phase error of -3.5 degrees between the output ports of the combining/splitting hybrid when the hybrid input powers were equal. Tests were also performed to determine the bandwidth and gain/phase margin of the feedback loops involved in the parallel-klystron controls. No evidence of feedback loop instability was noted. The bandwidth of the amplitude-control loop was limited to approximately $350 \mathrm{~Hz}$ due to the response time of the klystron anode modulator, and the phase loop bandwidth was limited to approximately $2 \mathrm{kHz}$ by the bandwidth of the vector voltmeter analog output.

In December 1999, $50 \mathrm{~mA}$ of beam was successfully stored utilizing RF1 and RF4 operating in parallel mode and RF2 operating in single-ended mode. The total storage-ring $\mathrm{rf}$ gap-voltage was being regulated against the effects of beam loading by two analog gap-voltage automatic gain control (AGC) feedback loops, one regulating the combined rf output of RF1 and RF4 in parallel, and the other regulating the rf output of RF2 in single-ended mode. The gap-voltage AGC loop controlled the rf output of the "master" parallel station (RF1) and the single-ended rf station (RF3) by varying mod-anode voltage. The rf output of the "slave" parallel rf station was controlled by the parallel-klystron amplitude-control loop, which maintained proper power balance at the input of the combining/splitting hybrid.

\section{CONCLUSION AND FUTURE OPERATING PLAN}

The parallel-klystron operation scheme at APS has been shown to meet the phase- and amplitude-control requirements for storage-ring beam operations at APS. The design utilizes existing APS low-level rf hardware and implementation requires minimum modification to existing systems. It has proven to be compatible with existing APS klystron HVPS systems and other highpower rf components. Further system tests, plus improvements in the system hardware and development of customized EPICS operating screens for parallel operation, are scheduled for the summer of 2001 in preparation for planned high-current runs in the fall of 2001.

\section{REFERENCES}

[1] Y.W. Kang and D. Horan, "Reconfigurable HighPower RF Systems in the APS," Proc. 1997 Particle Accelerator Conf., pp. 2935-2937 (1997).

[2] D. Horan and E. Cherbak, "An Analog RF Gap Voltage Regulation System for the Advanced Photon Source Storage Ring," Proc. 1999 Particle Accelerator Conf., pp. 1019-2021 (1999). 